\title{
Tip Recycling for Atomic Force Microscopy-Based Tip-Enhanced Raman Spectroscopy
}

\section{Journal Article}

Author(s):

Bartolomeo, Giovanni L.; Goubert, Guillaume (D); Zenobi, Renato (D)

Publication date:

2020-11

Permanent link:

https://doi.org/10.3929/ethz-b-000406179

Rights / license:

In Copyright - Non-Commercial Use Permitted

Originally published in:

Applied spectroscopy 74(11), https://doi.org/10.1177/0003702820916234

Funding acknowledgement:

741431 - Nanoscale Vibrational Spectroscopy of Sensitive 2D Molecular Materials (EC) 


\title{
Tip Recycling for Atomic Force Microscopy-Based Tip- Enhanced Raman Spectroscopy
}

\author{
Giovanni Luca Bartolomeo1, Guillaume Goubert1, ${ }^{*}$, and Renato Zenobi1, ${ }^{*}$ \\ ${ }_{1}$ Department of Chemistry and Applied Biosciences, Swiss Federal Institute of \\ Technology (ETH) Zurich, Vladimir-Prelog-Weg 1-5 8093 Zurich (Switzerland) \\ Corresponding authors: goubert@org.chem.ethz.ch, zenobi@org.chem.ethz.ch
}

\begin{abstract}
Tip-Enhanced Raman Spectroscopy (TERS) is a powerful tool for the characterization of surfaces and 2D materials, delivering both topographical and chemical information with nm-scale spatial resolution. AFM-TERS combines an atomic force microscope (AFM) with a Raman spectrometer and is a very versatile technique, capable of working in vacuum, air and liquid, and on a variety of different samples. A metalized AFM tip is necessary in order to take advantage of the plasmonic enhancement. The most commonly used metal is Ag, thanks to its high plasmonic activity in the visible range. Unfortunately, though, the tip metallization process is still challenging and not fully reliable, yielding inconsistent enhancement factors even within the same batch of tips; as a consequence, many tips are usually prepared at once (for a single experiment), to ensure that at least one of them is sufficiently active. As the lifetime of an unprotected, Ag-coated plasmonic probe is only a few hours, the procedure is inefficient and results in a substantial waste of materials and money. In this work, we establish a cleaning routine to effectively re-use Ag-coated AFM-TERS probes, drastically reducing costs without compromising the quality of the experimental results.
\end{abstract}

\section{Introduction}

Tip-enhanced Raman spectroscopy (TERS) is an analytical technique which combines scanning probe microscopy (such as Scanning Tunneling Microscopy - STM or Atomic Force Microscopy - AFM) with Raman spectroscopy with the aim of obtaining a vibrational spectrum from very small spots of the sample, well below the optical diffraction limit. It was independently demonstrated by four different 
laboratories in 20001-4 and has proven very effective for nanoscale characterization of surfaces; recently, a resolution as low as $1.7 \mathrm{~nm}$ was shown in ambient conditions5, while sub-molecular resolution was achieved in ultra-high vacuum6. STM-TERS has established itself as a valuable tool for the investigation of 2D materials 7 but requires the substrate to be conductive. Moreover, the sample thickness should allow the current to tunnel through (practically, $<5 \mathrm{~nm}$ )8. AFM-TERS, on the other hand, is more versatile, since it is neither substrate-sensitive nor limited by the sample thickness and can thus be used for a much wider variety of applications, e.g. the characterization of biological membranes or dielectric materials9. Experiments were successfully performed on lipids 10, DNA 11 and proteins12, even in a liquid environment13 with AFMTERS. The heart of every AFM-TERS experiment is an enhancing plasmonic probe; commercially available AFM cantilevers, usually made of $\mathrm{Si}$, need to be metalized to become suitable as TERS probes. Many tip fabrication methods have been investigated so far, including electrodeposition 14 , electroless deposition 15 or the attachment of Ag nanowires to the cantilever 16, but the most common functionalization method is the deposition of a thin layer of plasmonic metal (generally $\mathrm{Ag}$ or $\mathrm{Au}$ ) via physical vapor deposition (PVD). Despite being widespread and effective, the PVD coating process requires several hours and an expensive vacuum setup. Conversely, the average operational lifetime of an unprotected Ag-coated plasmonic tip is less than 5 hours in ambient conditions 17. Furthermore, as the plasmonic activity of probes can greatly vary (some tips may simply not be active at all), it is common practice for every experimental measurement to prepare 3 to 5 tips just as a backup. At the end of their life cycle, whether used or not, metalized tips are discarded as they are no longer enhancing and possibly contaminated with carbonaceous species from the environment. Considering their high cost (up to a hundred dollar each) and the long time it takes to prepare them, the current procedure is neither time nor economically efficient. The possibility to recycle tips is thus attractive from two points of view: first, reusing probes represents an obvious economic advantage; second, defining a consistent cleaning routine would possibly help tackling another major problem of TERS, i.e. environmental contamination. In fact, even though the technique developed dramatically in the last years, contamination from the environment, resulting in inconsistent and spurious spectra, is a key issue the TERS community has been facing since the earliest experiments and is yet to be fully resolved18. An acceptable procedure will allow the restoration the tip to a pristine condition, removing all the silver 
and leaving the Si structure intact, ready to be coated again. In this project, several approaches were investigated with the goal of establishing an effective workflow for the recycling of Ag-coated AFM-TERS tips. Selective removal of the metal coating was achieved, without compromising the underlying silicon structure. Recycled tips were studied using SEM and AFM and showed no significant difference in structural features and spatial resolution compared to brand new ones. Most importantly, TERS experiments on test samples exhibited no deviation from reference spectra, confirming the reliability of the approach. These results pave the way to a cheaper and more efficient probe preparation protocol that could easily be implemented in other laboratories.

\section{Results and Discussion}

First, different cleaning mixtures were tested in order to verify the effectiveness of $\mathrm{Ag}$ removal as well as their ability to leave the underlying Si structure untouched. A total of 7 cleaning baths were selected (Table 1) and each one of them was tested on 4 tips. Since $\mathrm{Ag}$ is a noble metal, the presence of an oxidizing agent was required in all the mixtures. The evaluation of the result was based on SEM experiments (secondary electrons (SE) for observing of the tip apex, backscattered electrons (BSE) for qualitative identification of residual $\mathrm{Ag}$ ) and on energy dispersive X-ray (EDX) spectra for the quantification of residual Ag. SEM images are available in supporting information (Figure S1).

\begin{tabular}{|c|c|c|c|}
\hline Cleaning mixture & Exp. Time (s) & Ag removal & Damage \\
\hline $18 \mathrm{M} \mathrm{H}_{2} \mathrm{SO}_{4}: 10 \mathrm{M} \mathrm{H}_{2} \mathrm{O}_{2}=3: 1$ & 30 & Low & High \\
\hline $9 \mathrm{M} \mathrm{H}_{2} \mathrm{SO}_{4}: 3 \mathrm{M} \mathrm{H}_{2} \mathrm{O}_{2}=3: 1$ & 60 & Low & High \\
\hline $16 \mathrm{M} \mathrm{HNO}_{3}$ & 30 & High & High \\
\hline $4 \mathrm{M} \mathrm{HNO}_{3}$ & 60 & Low & Low \\
\hline $18 \mathrm{M} \mathrm{NH}_{3}: 10 \mathrm{M} \mathrm{H}_{2} \mathrm{O}_{2}=3: 1$ & 30 & High & High \\
\hline $4 \mathrm{M} \mathrm{NH}_{3}, 1 \mathrm{M} \mathrm{H}_{2} \mathrm{O}_{2}$ & 1800 & High & Low \\
\hline $\mathrm{O}_{3}+4 \mathrm{M} \mathrm{NH}_{3}, 1 \mathrm{M} \mathrm{H}_{2} \mathrm{O}_{2}$ & $900+30$ & High & None \\
\hline
\end{tabular}

Table 1: Mixtures selected for the cleaning process. Composition, exposure time and performances of all the protocols are presented here. 
Piranha solution etching ( $\left.18 \mathrm{M} \mathrm{H}_{2} \mathrm{SO}_{4}: 10 \mathrm{M} \mathrm{H}_{2} \mathrm{O}_{2}=3: 1\right)$ is well known as a fast and effective way to remove residual traces of organic contaminations (e.g. photoresists) from surfaces 19. In our case, it would serve the double function of removing both $\mathrm{Ag}$ and organic contaminants from the Si surface, making the substrate ready for a second deposition. Unfortunately, the damage to the Si structure of the tip after just 30 seconds of exposure was considerable while the removal of Ag was very limited; for these reasons, the method cannot be considered effective (Figure 1). The degradation mechanism of $\mathrm{Si}$ (and of the silicon oxide layer on the surface) when exposed to sulfuric acid is not clear (acids should not be able to etch silicon) and, to the best of our knowledge, has never been reported in literature. A second cleaning attempt, using diluted piranha solution, showed again heavy damage to the tip surface, yet it was not able to completely remove the silver layer. Nitric acid appeared to be a good alternative considering its ability to dissolve silver ( $\mathrm{HNO}_{3}$ is an oxidizing acid) and was tested in two concentrations: $16 \mathrm{M}$ and $4 \mathrm{M}$. In the case of concentrated (16 M) nitric acid, the Si surface was severely compromised, but the coating layer was fully removed. On the other hand, diluting the acid was a "double-edged sword"; damage to the tip apex was reduced while the Ag removal efficiency was going down.

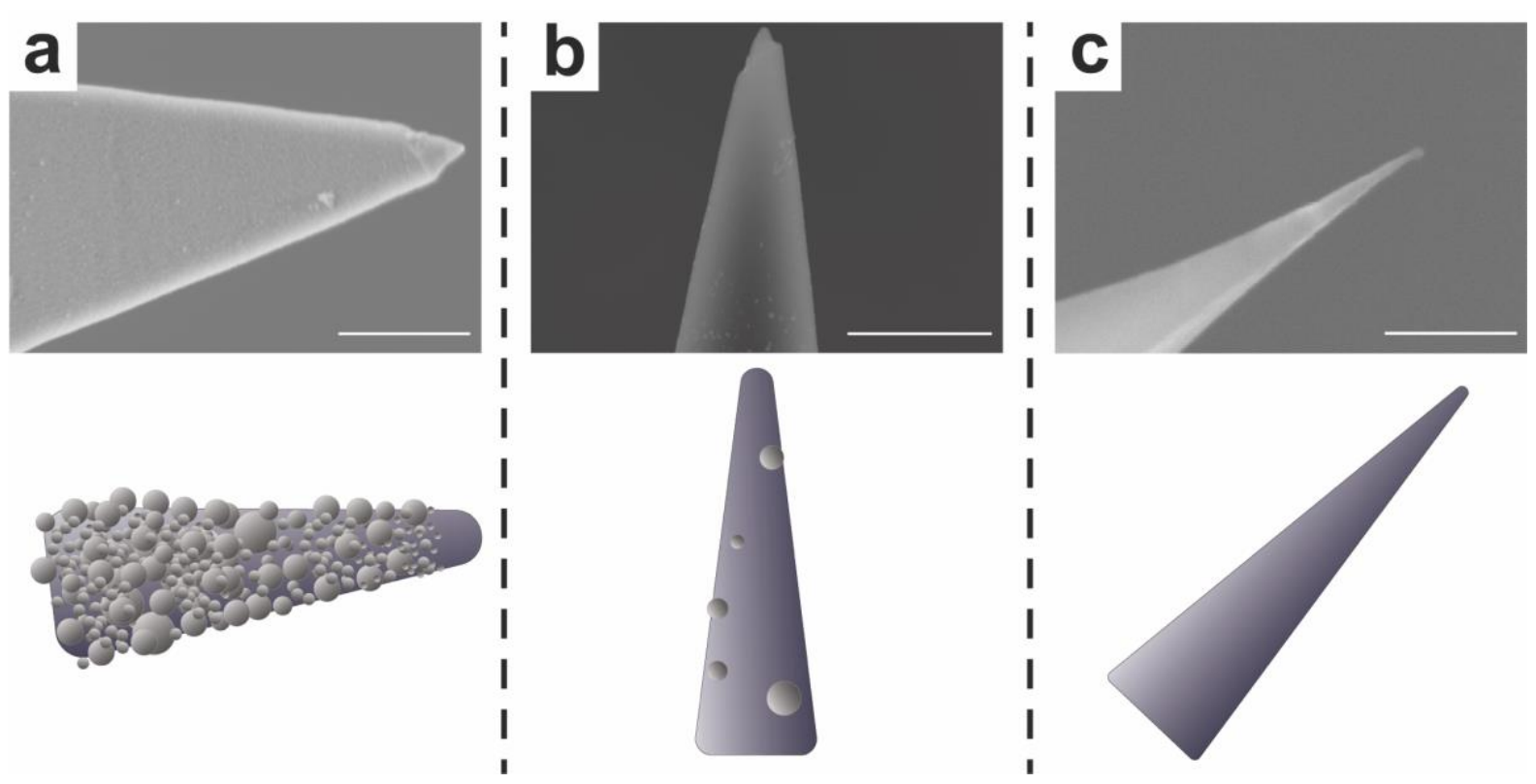

Figure 1: SEM images and schematic representation of Ag-coated AFM tips treated with: a) $18 \mathrm{M} \mathrm{H}_{2} \mathrm{SO}_{4}: 10 \mathrm{M} \mathrm{H}_{2} \mathrm{O}_{2}=3: 1$, b) $16 \mathrm{M} \mathrm{HNO}_{3}$ and c) $\mathrm{O}_{3}+4 \mathrm{M} \mathrm{NH}_{3}, 1 \mathrm{M} \mathrm{H}_{2} \mathrm{O}_{2}$. Silver removal efficiency is high in b) and c) as the surface is smooth and clean. On 
the other hand, aggressive chemicals can irreversibly damage the apex, as in a) and b). Treatment c) is the best compromise in terms of efficiency and selectivity. Scale bars: a) $2 \mu \mathrm{m}$, b) $3 \mu \mathrm{m}$, c) $300 \mathrm{~nm}$

"Base piranha" etching ( $18 \mathrm{M} \mathrm{NH}_{4} \mathrm{OH}: 10 \mathrm{M} \mathrm{H}_{2} \mathrm{O}_{2}=3: 1$ ) was tested then. It is strongly oxidizing, and the formation of the $\left[\mathrm{Ag}\left(\mathrm{NH}_{3}\right)_{2}\right]+$ complex helps solubilizing the silver quickly. However, the mixture is also very alkaline, favouring the dissolution of silica via the formation of silicilic acid $\left(\mathrm{H}_{4} \mathrm{SiO}_{4}\right)$ and silicates ( $\left.\mathrm{SiO}_{32}-\right)_{20}$. As a consequence, silver was removed very effectively but the tip apex was severely affected. Reducing the concentration of the species (using $4 \mathrm{M} \mathrm{NH}_{3}$ and $1 \mathrm{M} \mathrm{H}_{2} \mathrm{O}_{2}$ ) resulted in almost no damage to the silicon and in a lower efficiency of the removal process, again not an acceptable result. In order to obtain a quantitative removal, it was necessary to increase the exposure time to 1800 seconds (30'), but at this point silicon was being etched again. We decided to test a pre-oxidation step with ozone (15 minutes) to improve Ag removal. Exposing the tips to an ozone treatment before the wet chemistry process dramatically improved the performances of the subsequent cleaning, reducing the time required in the etching bath to 30 seconds, thus limiting the damage to the underlaying $\mathrm{Si}$ structure, from now on we refer to this procedure as Oz-BP (ozone + base piranha, last line in table 1). The SEM image acquired after Oz-BP shows that the apex is still sharp and undamaged while EDX spectroscopy confirms complete removal of $\mathrm{Ag}$ from the surface (Figure $\mathrm{S} 2$ ). The coating-cleaning process was repeated 3 times in a row on the same set of tips in order to study possible long-term damage of Oz-BP. Tips were analysed with SEM after every step to constantly monitor their structure. After each cleaning process, we observe that the Ag coating is quantitatively removed, but the tip is covered by an unknown contamination (Figure S3). BSE images clearly show the presence of carbon at the apex (Figure S4), most probably as a result of the electron beam of the microscope itself 21 . To confirm this hypothesis, the tips were imaged by SEM only at the beginning and at the end of a 3cycle round. As can be seen in Figure 2c-d, almost no deposition of carbon was observed and the tip apex appeared clean and sharp, proving the carbon contamination is an artefact of SEM imaging. 

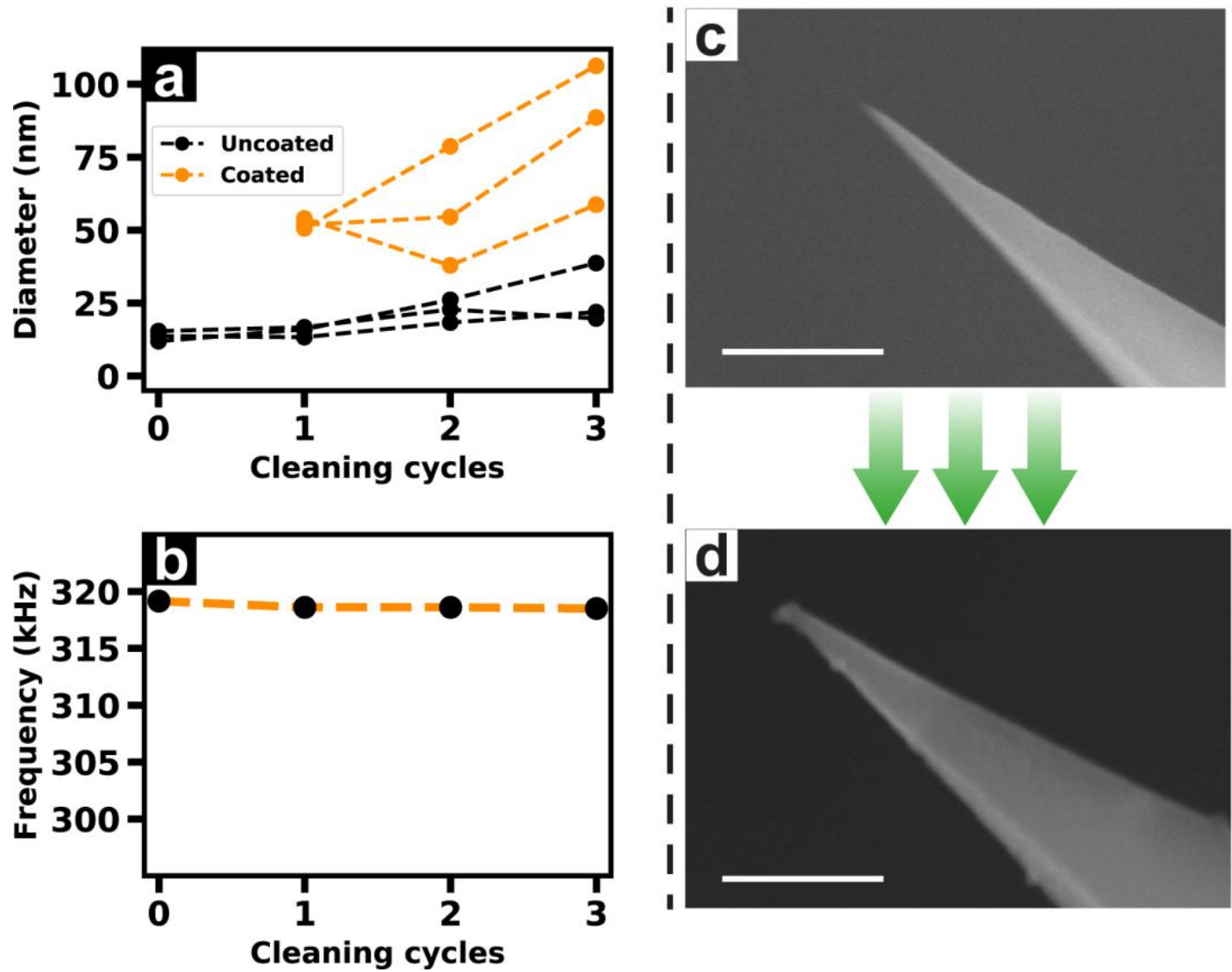

Figure 2: a) carbon build-up on the tip apex (see SI). The size of the tip apex tends to slightly increase as a result of carbon deposits. In any case, the diameter of a cleaned tip is always smaller than a coated one. By limiting high-energy electron exposure, this phenomenon can be significantly reduced: in this case, a tip is imaged just at the start (c) and after three full coating-cleaning cycles (d), showing almost no carbon deposition. In b) a limited shift of the resonance frequency of the tip is observed; this just happened at the end of the first cleaning cycle and may be due to the oxidation of the Si surface. Scale bars in c and d: $300 \mathrm{~nm}$.

Interestingly, after the first cleaning cycle the resonance frequency of the cantilever shifted to a slightly lower value (less than $1 \%$, see Figure $2 \mathrm{~b}$ ). This phenomenon occurred consistently on every tip tested. According to the equation (1), and assuming the elastic constant $\mathrm{k}$ did not change in the process, the resonance shift can be attributed to a slight mass (M) increase; a reasonable explanation is the oxidation of exposed silicon (the tips are not back-coated with metal). This would also explain why the phenomenon is only observed once, even if the tip undergoes three cleaning cycles. 


$$
v=\frac{1}{2 \pi} \sqrt{\frac{k}{M}}
$$

All probes were tested on a sample consisting of single-walled carbon nanotubes (SWCNTs) on template-stripped gold (TSAu). Nanotubes were correctly identified using their diameter ( $2 \mathrm{~nm}$, equal to their height in the AFM image) as a reference. Then the resolution, defined as full width at half maximum of their Gaussian profile, was recorded. Spatial resolution (in the plane of the surface) was generally lower than expected because of convolution effects (Figure 3); nonetheless, no worsening was observed, even after 3 cleaning cycles. These results confirm that our cleaning protocol does not damage the structure or the tip of the AFM cantilever.
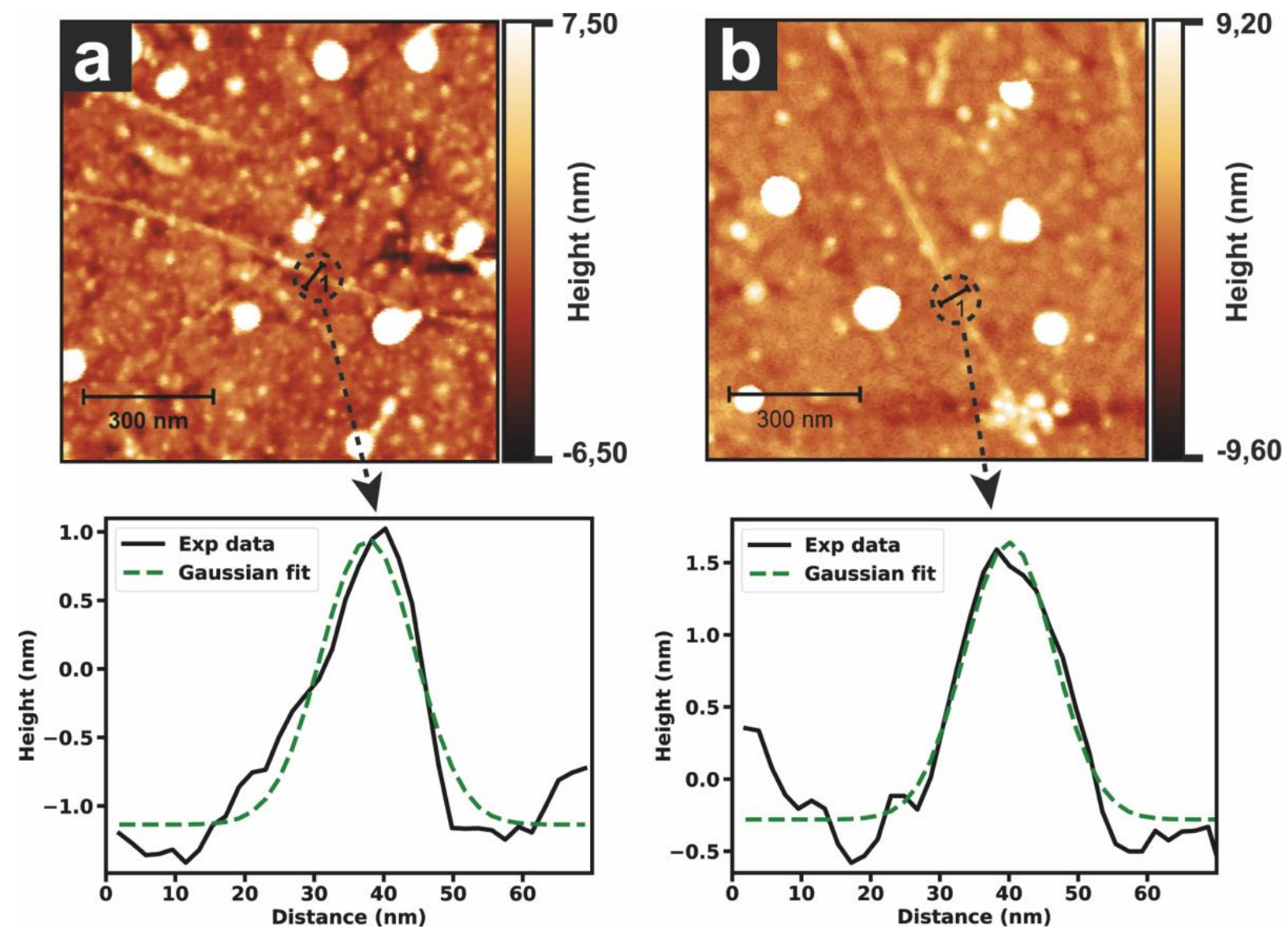

Figure 3: AFM height image and extracted profile of a SWCNT on TSAu with a brandnew tip (a) and with a recycled one (b). Even after three cleaning cycles the tip does not show any loss in resolution (FWHM for $a=22.5 \mathrm{~nm}$, FWHM for $b=21.4 \mathrm{~nm}$ ). 
Lastly, TERS was tested using the recycled tips. As reference sample, a 4-biphenylthiol (4-BPT) self-assembled monolayer (SAM) on TSAu was used. The activity of a set of 4 tips was tested throughout three recycling cycles (4 acquisitions in total). The activity of tips was evaluated by measuring the signal-to-noise ratio between the peak at $1079 \mathrm{~cm}-1$ (in-plane scissoring of $\mathrm{C}-\mathrm{H}$ bond) and the standard deviation of the noise in the region $1730-1880 \mathrm{~cm}-1$, according to the following formula (2):

$$
S / N=\frac{I_{\text {tip }(n)}}{R M S_{\text {noise }}}
$$

Where $\mathrm{S} / \mathrm{N}$ is the signal-to-noise ratio, Itip(n) is the intensity with a specific tip at 1079 $\mathrm{cm}-1, \mathrm{RMS}_{\text {noise is }}$ the root mean square of the noise, acquired between 1730 and 1880 $\mathrm{cm}-1$. The $1079 \mathrm{~cm}-1$ band was selected because it is specific for 4-BPT and is located in a clean region of the spectrum, while the noise was acquired in a silent region of the spectrum. The peak was fitted with a Gaussian function to obtain the peak intensity. Since a 4-BPT SAM does not exhibit any far-field spectrum, all the signal collected was considered as resulting from near-field. In Figure 4a an AFM-TERS tip was used to measure a spectrum $(10 \mathrm{~s}, 50 \mu \mathrm{W})$ in every phase of the process. The first deposition yielded a weakly enhancing tip, while in the second deposition the tip was almost non-active. However, the tip resulted active and strongly enhancing after the third and fourth coating. The three other tips prepared in the same batch (Figure $4 b)$, all follow the same trend. 

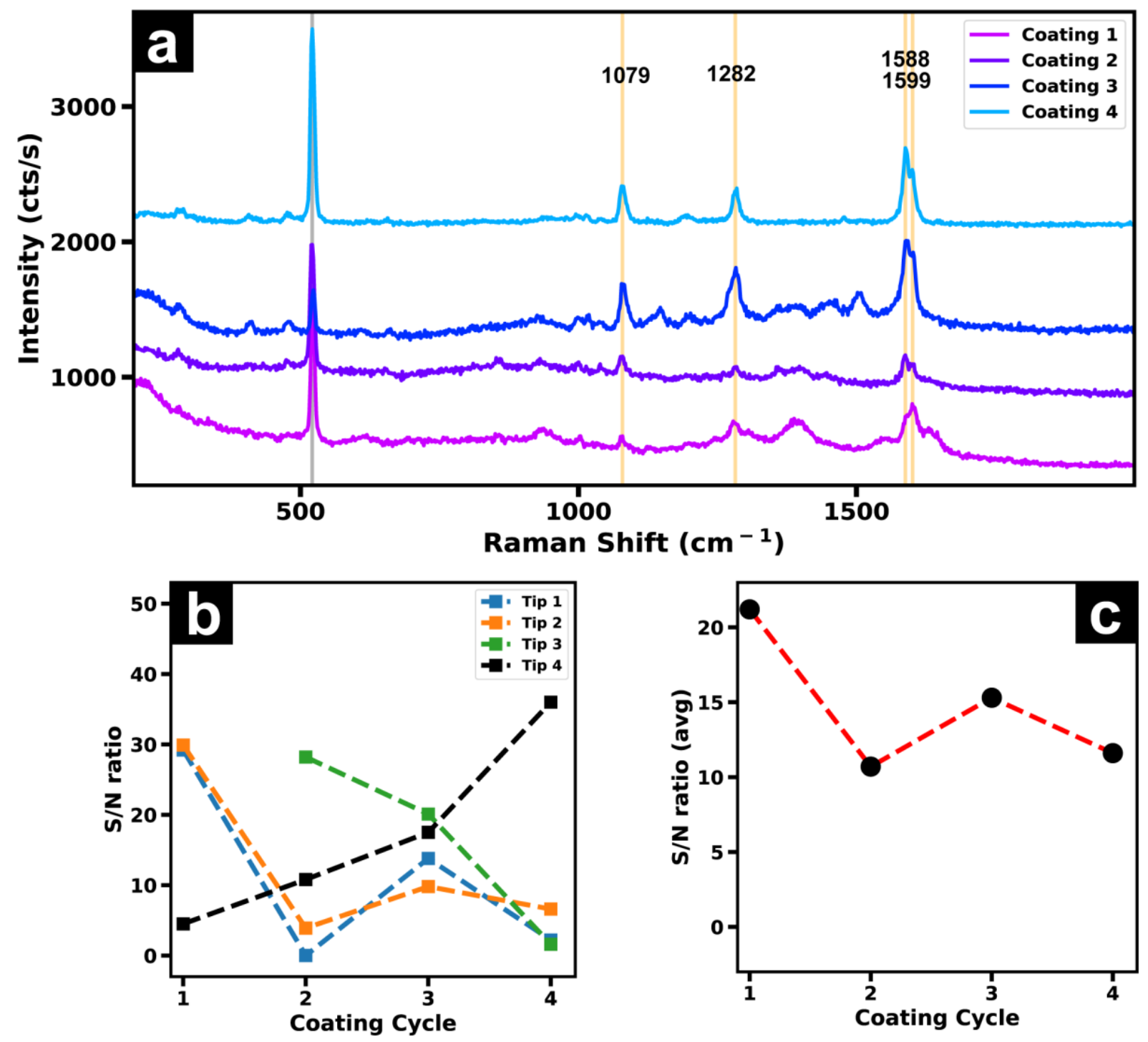

Figure 4: a) TERS spectra of a 4-biphenylthiol self-assembled monolayer acquired with the same AFM probe coated four different times. The characteristic bands of 4BPT $(1079,1282,1588$ and $1599 \mathrm{~cm}-1)$ are highlighted. The peak of silicon $(521 \mathrm{~cm}$ 1) is also highlighted and used to calibrate the spectrum. b) Quantification of the peak area (for the $1079 \mathrm{~cm}-1$ peak) for four tips after each coating step; despite a very high variability among different tips, recycled probes could be as good as (or potentially better) than new ones. c) Average peak area per coating cycle; the coating process seems to play a major role in defining the enhancing capability of probes.

This result clearly shows that there is no correlation between the number of recyclings and the near field signal, which varies greatly among tips. This finding is in full agreement with what one experiences with new AFM tips, as quite some variability is always observed. However, analysing the average $\mathrm{S} / \mathrm{N}$ of the four tips per coating 
step, a correlation is apparent; when one probe is strongly enhancing, all other probes of the same batch tend to be active. Considering the limited amount of data, one can only speculate that the defining step of the process is the metal deposition. A small change during this phase (e.g. operating pressure of the coating chamber, position of the metal source relative to the tips...) could affect all the tip of the same batch.

Even when recycled tips show carbon contaminations, as visible in Figure 4a, coating 3 , the number of recyclings does not correlate with the intensity of carbon contaminations signals. In some cases, the opposite may be true, as seen in Figure S5, where the spurious signal arising from contamination decreased throughout the cleaning/coating cycles (from 1 to 4 ) and the spectrum of 4-BPT become better defined. It could be due to the strong oxidizing power of the Oz-BP procedure. This calls for a more detailed study of the effect of recycling on contamination in ambient TERS, which is out of the scope of the work presented here.

\section{Conclusion}

We successfully implemented a protocol for recycling used Ag-coated AFM-TERS tips. By selecting the best wet-chemistry approach, rapid and effective removal of the silver coating was achieved, while damage to the silicon tip was avoided. Most importantly, recycled tips perform as well as brand new ones in TERS experiments, making it possible for laboratories to make better use of their resources, and perform AFM-TERS experiments more efficiently.

\section{Experimental section}

Sulfuric acid (98\%), nitric acid (65\%), hydrogen peroxide (30\%), ammonium hydroxide (35\%), and 4-biphenyl thiol were purchased from Sigma Aldrich (St. Louis, MO, USA) and used without further purification. Solutions were diluted with distilled water. ATECNC cantilevers (NanoAndMore GmbH, Wetzlar, Germany) were used as test probes for all the experiments. A special holder was designed to facilitate the handling, coating, cleaning and imaging of the probes. To compensate for the angle of the tip, an offset of $39^{\circ}$ was imposed by tilting the tip holder (further details are available in SI, Figure S6). Tips were coated with silver (99.95\%, Alfa Aesar, Haverhill, MA, USA) up to $35 \mathrm{~nm}$ nominal thickness via physical vapor deposition at a pressure $<10-6$ mbar. For this purpose, a Baltec MCS 010 evaporator equipped with a Baltec QSG 060 
quartz balance was used. The UV-O3 cleaner used in these experiments was from Ossila Ltd, Sheffield, UK. Scanning electron microscope images were acquired using a Hitachi SU5000 or SU8200 (Hitachi Ltd., Tokyo, Japan) instrument. Unless otherwise specified, for secondary electron (SE) imaging, an acceleration voltage of $10 \mathrm{kV}$ and a current intensity of $5 \mathrm{nA}$ was applied, whereas for backscattered electron (BSE) imaging, the acceleration voltage was increased to $15 \mathrm{kV}$ and the current intensity to $30 \mathrm{nA}$. For energy-dispersive X-ray spectroscopy (EDX), a Hitachi SU8200 (Hitachi Ltd., Tokyo, Japan) instrument was used: the voltage was set to $10 \mathrm{kV}$, the current intensity to $50 \mathrm{nA}$, and the signal was acquired with a X-MaxN 80T (Oxford Instruments plc, Abingdon, UK) detector. AFM data were acquired on a Bioscope Catalyst (Bruker Corporation, Billerica, MA, USA). Both AFM and SEM images were processed with Gwyddion software (gwyddion.net). TERS experiments were performed on a Ntegra Spectra II (NT-MDT Spectrum Instruments, Zelenograd, Russia), equipped with a $632.8 \mathrm{~nm} \mathrm{He}-\mathrm{Ne}$ laser. Spectral data were analysed and plotted with a custom-made Python routine. Additional etails about procedures and preparation of the samples could be found in SI.

\section{Notes}

The original data used in this publication are made available in a curated data archive at ETH Zurich (https://www.research-collection.ethz.ch) under the DOI 10.3929/ethzb-000396976

\section{Acknowledgements}

The authors would like to thank ScopeM for the SEM instrumentation. Special thanks to Christian Marro from our mechanical workshop for his substantial help. GLB gratefully acknowledge the Scholarship Fund of Swiss Chemical Industry (SSCl) for financial support. This project is co-financed by the European Union through the ERC grant N. 741431 2D-NanoSpec.

\section{References}

1. R.M. Stöckle, Y.D. Suh, V. Deckert, R. Zenobi. "Nanoscale chemical analysis by tip-enhanced Raman spectroscopy”. Chem. Phys. Lett. 2000. 318(1-3): 131-136. 10.1016/S00092614(99)01451-7. 
2. M.S. Anderson. "Locally enhanced Raman spectroscopy with an atomic force microscope". Appl. Phys. Lett. 2000. 76(21): 3130-3132. 10.1063/1.126546.

3. B. Pettinger, G. Picardi, R. Schuster, G. Ertl. "Surface Enhanced Raman Spectroscopy: Towards Single Molecule Spectroscopy”. Electrochemistry. 2000.

4. N. Hayazawa, Y. Inouye, Z. Sekkat, S. Kawata. "Metallized tip amplification of near-field Raman scattering”. Opt. Commun. 2000. 183(1): 333-336. 10.1016/S0030-4018(00)00894-4.

5. C. Chen, N. Hayazawa, S. Kawata. "A $1.7 \mathrm{~nm}$ resolution chemical analysis of carbon nanotubes by tip-enhanced Raman imaging in the ambient". Nat. Commun. Nature Publishing Group, a division of Macmillan Publishers Limited. All Rights Reserved., 2014. 5: 3312.

6. X. Chen, P. Liu, Z. Hu, L. Jensen. "High-resolution tip-enhanced Raman scattering probes submolecular density changes”. Nat. Commun. Springer US, 2019. 10(1). 10.1038/s41467-01910618-x.

7. F. Shao, R. Zenobi. "Tip-enhanced Raman spectroscopy: principles, practice, and applications to nanospectroscopic imaging of 2D materials". Anal. Bioanal. Chem. Analytical and Bioanalytical Chemistry, 2019. 411(1): 37-61. 10.1007/s00216-018-1392-0.

8. A.F. Raigoza, J.W. Dugger, L.J. Webb. "Review: Recent advances and current challenges in scanning probe microscopy of biomolecular surfaces and interfaces". ACS Appl. Mater. Interfaces. 2013. 5(19): 9249-9261. 10.1021/am4018048.

9. L. Gao, H. Zhao, T. Li, P. Huo, D. Chen, B. Liu. "Atomic force microscopy based tip-enhanced Raman spectroscopy in biology". Int. J. Mol. Sci. 2018. 19(4). 10.3390/ijms19041193.

10. L. Opilik, T. Bauer, T. Schmid, J. Stadler, R. Zenobi. "Nanoscale chemical imaging of segregated lipid domains using tip-enhanced Raman spectroscopy". Phys. Chem. Chem. Phys. 2011. 13(21): 9978. 10.1039/c0cp02832k.

11. E. Lipiec, R. Sekine, J. Bielecki, W.M. Kwiatek, B.R. Wood. "Molecular characterization of DNA double strand breaks with tip-enhanced Raman scattering". Angew. Chemie - Int. Ed. 2014. 53(1): 169-172. 10.1002/anie.201307271.

12. E. Lipiec, D. Perez-Guaita, J. Kaderli, B.R. Wood, R. Zenobi. "Direct Nanospectroscopic Verification of the Amyloid Aggregation Pathway". Angew. Chemie - Int. Ed. 2018. 57(28): 85198524. 10.1002/anie.201803234.

13. A. Nakata, T. Nomoto, T. Toyota, M. Fujinami. "Tip-enhanced Raman spectroscopy of lipid bilayers in water with an alumina- and silver-coated tungsten tip." Anal. Sci. 2013. 29(9): 8659. 10.2116/analsci.29.865.

14. T.X. Huang, C.W. Li, L.K. Yang, J.F. Zhu, X. Yao, C. Liu, et al. "Rational fabrication of silvercoated AFM TERS tips with a high enhancement and long lifetime". Nanoscale. Royal Society of Chemistry, 2018. 10(9): 4398-4405. 10.1039/c7nr08186c.

15. P.R. Brejna, P.R. Griffiths. "Electroless deposition of silver onto silicon as a method of preparation of reproducible surface-enhanced Raman spectroscopy substrates and tipenhanced raman spectroscopy tips". Appl. Spectrosc. 2010. 64(5): 493-499. $10.1366 / 000370210791211682$. 
16. P. Walke, Y. Fujita, W. Peeters, S. Toyouchi, W. Frederickx, S. De Feyter, et al. "Silver nanowires for highly reproducible cantilever based AFM-TERS microscopy: Towards a universal TERS probe". Nanoscale. Royal Society of Chemistry, 2018. 10(16): 7556-7565. 10.1039/c8nr02225a.

17. N. Kumar, S.J. Spencer, D. Imbraguglio, A.M. Rossi, A.J. Wain, B.M. Weckhuysen, et al. "Extending the plasmonic lifetime of tip-enhanced Raman spectroscopy probes". Phys. Chem. Chem. Phys. 2016. 18(19): 13710-13716. 10.1039/C6CP01641C.

18. T. Deckert-Gaudig, A. Taguchi, S. Kawata, V. Deckert. "Tip-enhanced Raman spectroscopyfrom early developments to recent advances". Chem. Soc. Rev. Royal Society of Chemistry, 2017. 46(13): 4077-4110. 10.1039/c7cs00209b.

19. J.J. Cras, C.A. Rowe-Taitt, D.A. Nivens, F.S. Ligler. "Comparison of chemical cleaning methods of glass in preparation for silanization". Biosens. Bioelectron. 1999. 14(8-9): 683-688. 10.1016/S0956-5663(99)00043-3.

20. F.K. Crundwell. "On the Mechanism of the Dissolution of Quartz and Silica in Aqueous Solutions". ACS Omega. 2017. 2(3): 1116-1127. 10.1021/acsomega.7b00019.

21. M.T. Postek, A.E. Vladár, K.P. Purushotham. "Does your SEM really tell the truth? How would you know? Part 2". Scanning. 2014. 36(3): 347-355. 10.1002/sca.21124. 